\title{
Broadband 10Gb/s Graphene Electro-Absorption Modulator on Silicon for Chip-Level Optical Interconnects
}

\author{
Y. T. Hu*, M. Pantouvaki", S. Brems", I. Asselberghs", C. Huyghebaert", M. Geisler", C. Alessandri", \\ R. Baets", P. Absil", D. Van Thourhout" and J. Van Campenhout" \\ "IMEC, Kapeldreef 75, 3001 Leuven, Belgium \\ "Ghent University - IMEC, Photonics Research Group, Gent, Belgium \\ Tel: (32) 1628 7732, Email: Joris.VanCampenhout@imec.be
}

\begin{abstract}
We report the first silicon integrated graphene optical electro-absorption modulator capable of $10 \mathrm{~Gb} / \mathrm{s}$ modulation speed. We demonstrate low insertion loss and low drive voltage combined with broadband and athermal operation in a compact hybrid graphene-Si device, outperforming $\mathrm{Si}(\mathrm{Ge})$ optical modulators for future chip-level optical interconnect application.
\end{abstract}

\section{Introduction}

Integrated optical modulators with high modulation speed, small footprint and broadband, athermal operation are highly desired to realize high-density low-power optical interconnects tightly integrated with high-performance logic [1-2]. Silicon optical modulators have been strongly optimized over the past few years, but still suffer from large footprint [3], a narrow optical bandwidth or poor temperature tolerance [4]. Alternatively, compact broadband $\mathrm{SiGe}$ electro-absorption modulators (EAM) have been demonstrated, but with limited thermal stability [5]. Graphene, which exhibits $2.3 \%$ absorption over a wide spectral range from the visible to the infrared owing to its unique linear and gapless band dispersion, is a promising material for robust electro-absorption modulators [6-7]. In this paper, we demonstrate a broadband, athermal and compact graphene-Si EAM operating at $10 \mathrm{~Gb} / \mathrm{s}$, challenging $\mathrm{Si}(\mathrm{Ge})$ modulators for thermally volatile and area sensitive applications such as chip-level optical interconnects.

\section{Device Design and Fabrication}

The graphene EAM consists of a graphene-oxide-silicon (GOS) capacitor structure implemented on top of a planarized silicon-on-insulator (SOI) rib waveguide, as shown in Fig. 1. The graphene sheet interacts strongly with the guided optical modes of the sub-micron Si waveguide, and the optical loss can be modulated by controlling the Fermi level in the graphene layer through metal contacts placed on the graphene and Si layer. A modified 130nm CMOS shallow-trench isolation (STI) module with two $\mathrm{Si}$ patterning steps was used to define the Si rib waveguides on $200 \mathrm{~mm}$ SOI wafers. After planarization, three phosphorous ion implantation steps were carried out locally in the Si layer to reduce device resistance. Next, a $5 \mathrm{~nm}$ thick gate oxide was thermally grown on top of the $\mathrm{Si}$ waveguide and the wafer was diced. Subsequently, a single-layer graphene sheet grown by chemical vapor deposition (CVD) on a $\mathrm{Si} / \mathrm{SiO}_{2} / \mathrm{Cu}$ substrate was transferred to the Si chips using an elastomer stamp [8]. The graphene was patterned by photolithography followed by an oxygen plasma etch. Two separate steps defined the graphene-metal contact $(10 \mathrm{~nm} \mathrm{Cr} / 50 \mathrm{~nm}$ $\mathrm{Au} / 20 \mathrm{~nm} \mathrm{Ti} / 420 \mathrm{~nm} \mathrm{Au})$ and the Si-metal contact $(20 \mathrm{~nm}$ Ti/480nm Au). SEM images of a fabricated device are shown in Fig. 1. Raman data (Fig. 2) indicates that the single layer graphene quality is preserved after processing. Grating couplers [9] were implemented for fiber-to-chip optical coupling.
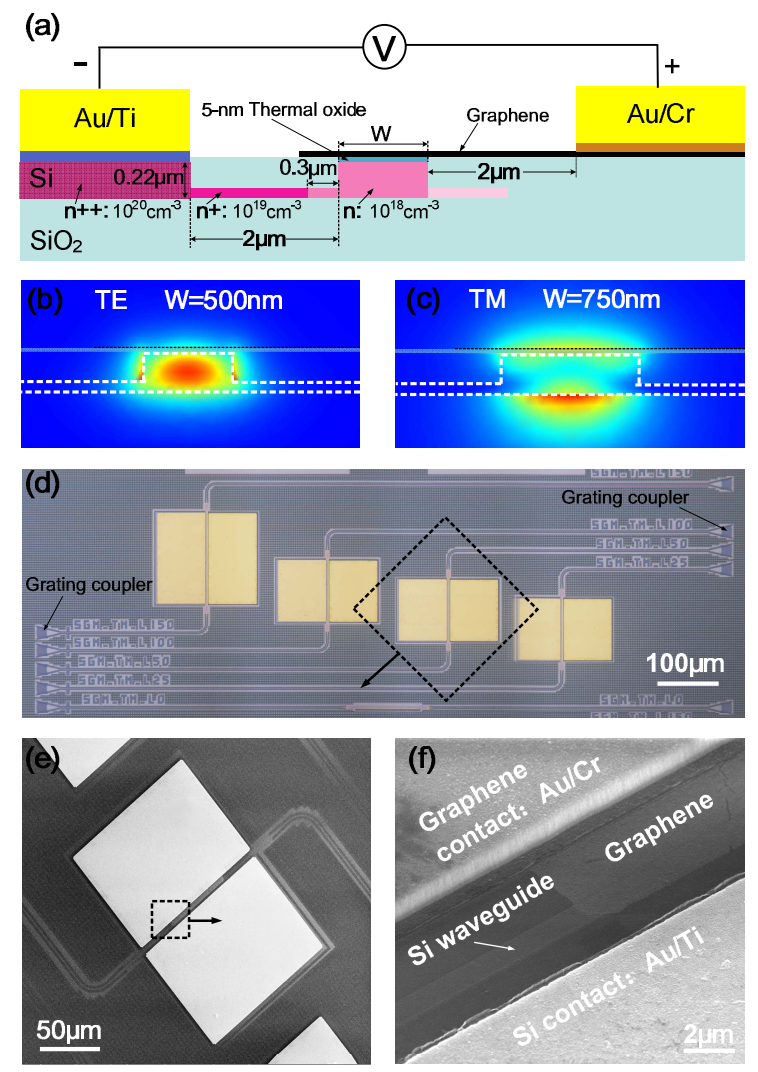

Fig. 1. Schematic cross section of the hybrid single-layer graphene-Si EAM showing the key structural parameters (a). Simulated optical mode profiles for hybrid graphene-Si waveguides designed for quasi TE (W $=500 \mathrm{~nm}$ ) (b) and $\mathrm{TM}(\mathrm{W}=750 \mathrm{~nm})$ polarized guided modes $(\mathrm{c})$. Optical microscope image showing the graphene EAM test site (d), and top-view (e) and tiltedview (f) SEM images of fabricated devices. 


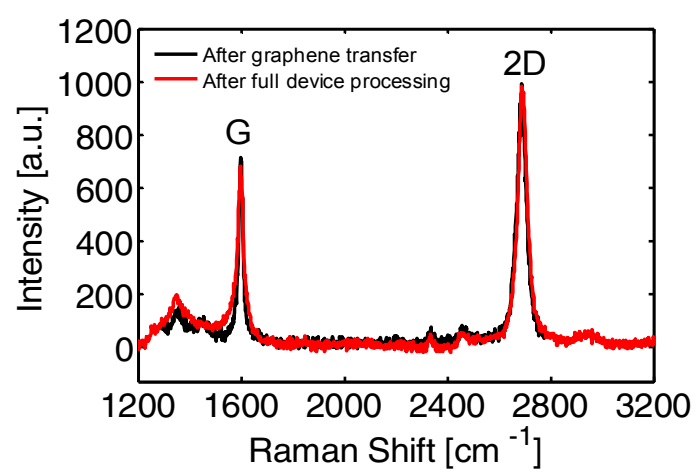

Fig. 2. Raman spectra of the single-layer graphene sheet after transfer onto the SOI chip and after full device processing. The intensity ratios of the D and $\mathrm{G}$ peak $\left(\mathrm{I}_{\mathrm{D}} / \mathrm{I}_{\mathrm{G}} \sim 0.3\right)$, as well as the $2 \mathrm{D}$ and $\mathrm{G}$ peak $\left(\mathrm{I}_{2 \mathrm{D}} / \mathrm{I}_{\mathrm{G}} \sim 1.4\right)$ indicate that the graphene quality is maintained after processing.
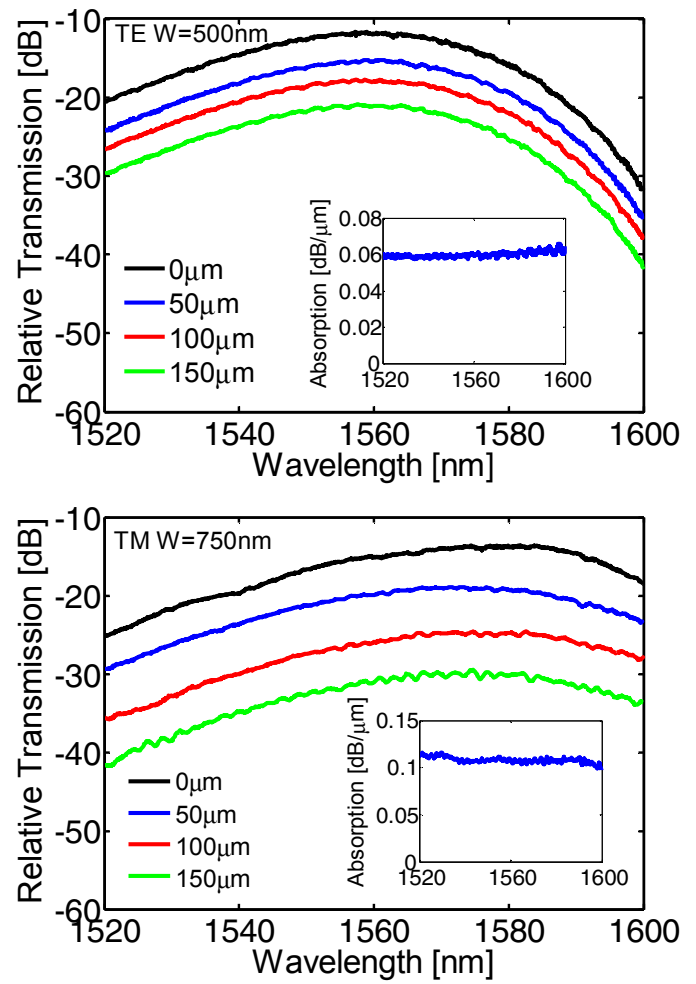

Fig. 3. Fiber-to-fiber transmission spectra for unbiased TE and TM graphene EAMs with graphene lengths of $50 \mu \mathrm{m}, 100 \mu \mathrm{m}, 150 \mu \mathrm{m}$, as well as for reference waveguides without graphene. Graphene-induced waveguide losses are extracted to be $0.06 \mathrm{~dB} / \mu \mathrm{m}$ for the TE mode and $0.11 \mathrm{~dB} / \mu \mathrm{m}$ for the TM mode across the full wavelength range (insets). The Gaussian-like roll-off of the transmission at short and long wavelengths originates from the grating coupler pair used for fiber-chip coupling [9].

\section{Static Performance}

Transmission spectra of unbiased graphene EAM devices with lengths of $50 \mu \mathrm{m}, 100 \mu \mathrm{m}, 150 \mu \mathrm{m}$ and reference waveguides without graphene are plotted in Fig. 3, for quasitransverse electric (TE) and quasi-transverse magnetic (TM) guided modes. Graphene-induced optical absorption losses of $\sim 0.06 \mathrm{~dB} / \mu \mathrm{m}$ and $\sim 0.11 \mathrm{~dB} / \mu \mathrm{m}$ are extracted for the TE and TM modes respectively over a wavelength range of $80 \mathrm{~nm}$ (limited by the grating couplers) around 1560nm (Fig. 3). By applying drive voltages $\mathrm{V}_{\mathrm{D}}$ in the range $-3 \mathrm{~V}$ to $3 \mathrm{~V}$, an extinction ratio (ER) of $\sim 5 \mathrm{~dB}$ and on-state insertion loss (IL) below $4 \mathrm{~dB}$ are obtained for a $50 \mu \mathrm{m}$ TM graphene EAM across the entire wavelength range (Fig. 4), owing to the strong interaction of the TM mode with the graphene sheet (Fig 1). Longer devices enable higher ER, exceeding 10dB for a $150 \mu \mathrm{m}$ device (Fig. 5), at the expense of higher IL. All tested graphene EAM devices exhibited strongest extinction at reverse drive voltages, likely caused by a Fermi-level shift in the graphene sheet due to doping. No significant changes in device response were observed for temperatures in the range $20^{\circ} \mathrm{C}$ to $49^{\circ} \mathrm{C}$, implying robust athermal operation (Fig. 6). Fig. 7 shows the $\mathrm{C}-\mathrm{V}$ and $\mathrm{I}-\mathrm{V}$ curves of the graphene EAMs, illustrating GOS accumulation and depletion capacitance for the $50 \mu \mathrm{m}$ long device of $\sim 220 \mathrm{fF}$ and $\sim 100 \mathrm{fF}$ at $\mathrm{V}_{\mathrm{D}}=2 \mathrm{~V}$ and $-2 \mathrm{~V}$ respectively, and leakage currents in the nA range.

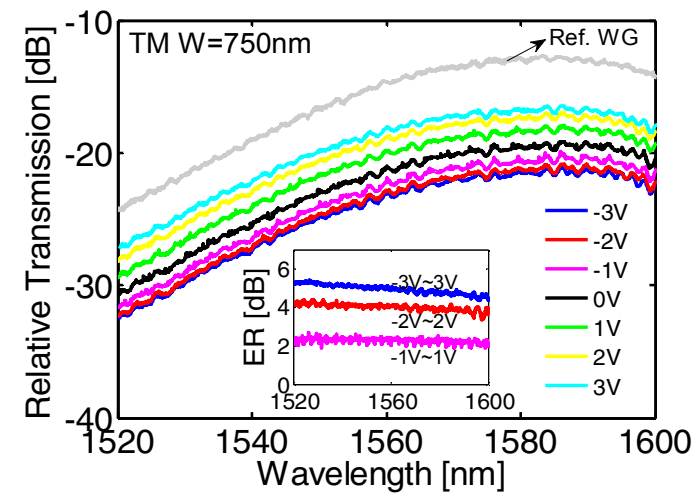

Fig. 4. Fiber-to-fiber transmission spectra of a $50 \mu \mathrm{m}$ TM graphene EAM at different drive voltages. An extinction ratio of $\sim 5 \mathrm{~dB}$ and insertion loss below $4 \mathrm{~dB}$ is obtained across the full wavelength range for $-3 \mathrm{~V}$ to $3 \mathrm{~V}$ voltage swing.

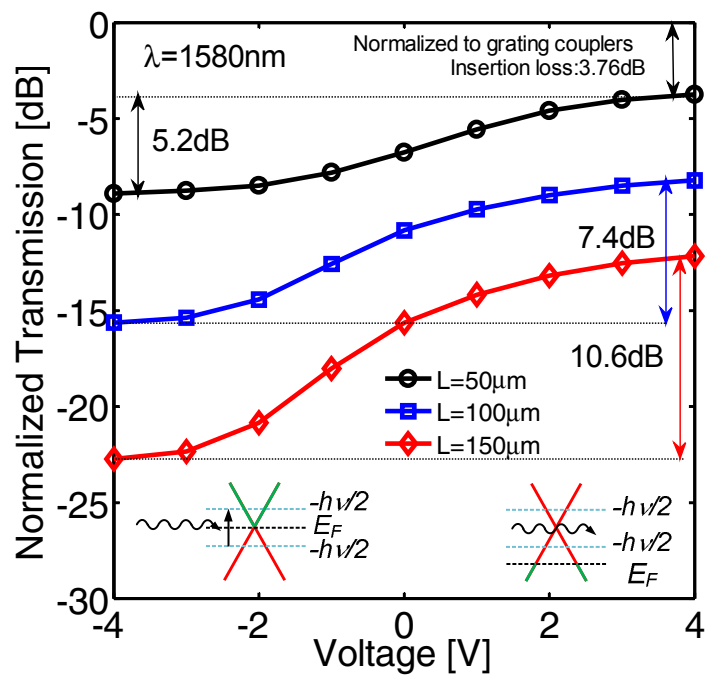

Fig. 5. Static switching curves versus applied voltage of TM graphene modulators with lengths of $50 \mu \mathrm{m}, 100 \mu \mathrm{m}$ and $150 \mu \mathrm{m}$ at $1585 \mathrm{~nm}$ wavelength, normalized for the optical insertion loss of the grating couplers. All EAM devices exhibit maximum absorption at $-4 \mathrm{~V}$, suggesting a graphene Fermi level close to the Dirac point, and strong absorption bleaching at $4 \mathrm{~V}$, suggesting strong hole accumulation $\left(\mathrm{E}_{\mathrm{F}}<-\mathrm{hv} / 2, \mathrm{~h}=\right.$ Planck's constant, $v=$ photon frequency). 


\section{High Speed Performance}

Radio-frequency (RF) S11 measurements and fitting to an electrical circuit model suggest a series resistance of Rs 250 and accumulation (depletion) capacitance of $\mathrm{CgSi} 200 \mathrm{fF}$ (80fF) for the 50um device (Fig. 8). The electrooptical S21 response shows RC-limited 3-dB bandwidths of $5.9 \mathrm{GHz}$ and $2.9 \mathrm{GHz}$ at reverse and forward bias respectively (Fig. 9), an up to 5 times improvement over earlier demonstrations [6-7], and in good agreement with the electrical model. Next, eye diagrams were generated for the $50 \mu \mathrm{m}$ graphene EAM with a drive voltage of $2.5 \mathrm{Vpp}$ and $1.75 \mathrm{~V}$ forward bias, using the setup shown in Fig 10. Wide open eye diagrams with dynamic ER $2.5 \mathrm{~dB}$ and low jitter are obtained at $6 \mathrm{~Gb} / \mathrm{s}$ and up to $10 \mathrm{~Gb} / \mathrm{s}$ (Fig. 11), across a $35 \mathrm{~nm}$ wavelength range (Fig. 12). This is the first demonstration of high-quality optical modulation at high bit rates by a graphene EAM. The energy consumption is estimated to be $\sim 350 \mathrm{fJ} / \mathrm{bit}\left(\mathrm{E}_{\mathrm{bit}} \sim \mathrm{CV}^{2} / 4\right)$. Finally, the performance metrics are compared to state-of-the-art $\mathrm{Si}(\mathrm{Ge})$ modulators in Table 2, illustrating the thermal, bandwidth and footprint benefits of the graphene EAM. Based on the electrical model, design optimizations are expected to reduce $\mathrm{R}_{\mathrm{s}}$ by a factor of $2-4$, targeting $25 \mathrm{~Gb} / \mathrm{s}$ modulation speed and beyond.

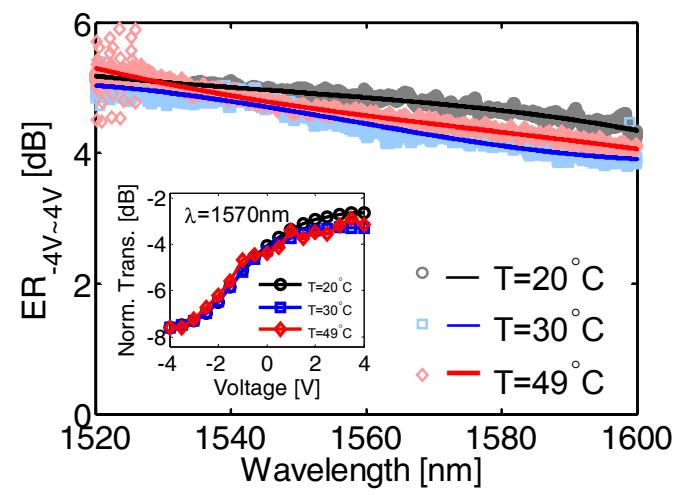

Fig. 6. Static extinction ratio versus wavelength of a $50 \mu \mathrm{m}$ TM graphene EAM measured for $-4 \mathrm{~V}$ to $4 \mathrm{~V}$ voltage swing at chip temperatures of $20^{\circ} \mathrm{C}$, $30^{\circ} \mathrm{C}$ and $49^{\circ} \mathrm{C}$. The inset shows the normalized static switching curves vs. applied voltage.

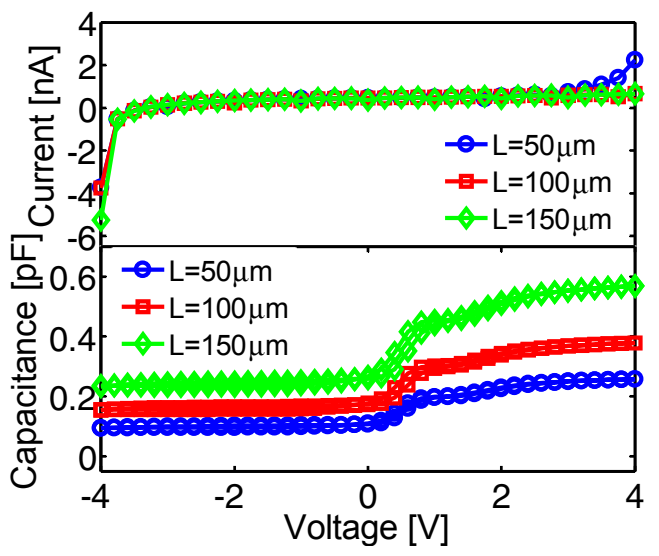

Fig. 7. Static I-V and quasi-static $(100 \mathrm{kHz}) \mathrm{C}-\mathrm{V}$ curves for TM graphene EAMs with lengths of $50 \mu \mathrm{m}, 100 \mu \mathrm{m}$ and $150 \mu \mathrm{m}$.

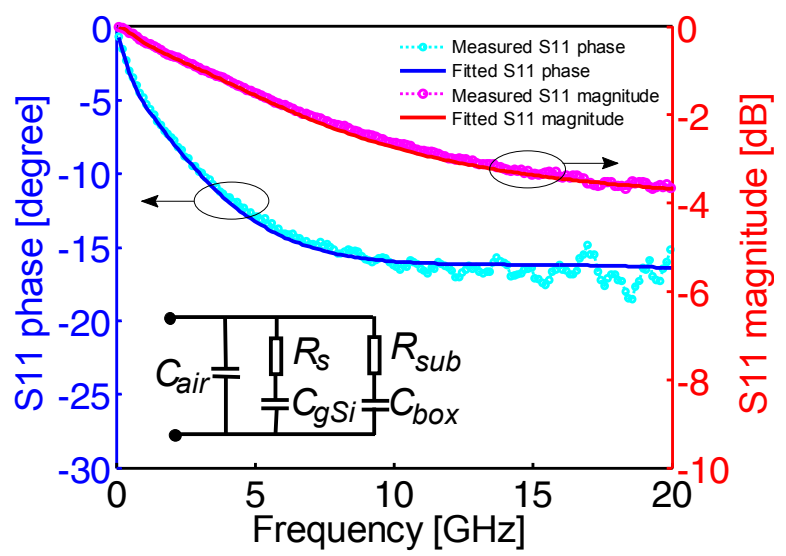

Fig. 8. Measured RF S11 response of the $50 \mu \mathrm{m}$ graphene EAM at $0 \mathrm{~V}$ bias and the fitted curves using an equivalent electrical circuit model (inset). $\left(\mathrm{C}_{\text {air }}\right.$ $=$ the capacitance between the two electrodes through the air, $\mathrm{R}_{\mathrm{s}}=$ the series resistance between the two electrodes, $\mathrm{C}_{\mathrm{gSi}}=$ the capacitance of the GOS structure, $R_{\text {sub }}=$ the resistance of the substrate, $C_{b o x}=$ the capacitance of the buried oxide layer).

Table 1. S11 fitting parameters of the $50 \mu \mathrm{m}$ graphene EAM for bias voltages of $0,-1$ and $2 \mathrm{~V}$, and electrical $3 \mathrm{~dB}$ RF bandwidth estimated from the fit.

\begin{tabular}{|c|c|c|c|c|c|c|}
\hline & $\mathbf{R}_{\mathbf{s}}(\boldsymbol{\Omega})$ & $\mathbf{C}_{\mathbf{g S i}}(\mathbf{f F})$ & $\mathbf{R}_{\text {sub }}(\boldsymbol{\Omega})$ & $\mathbf{C}_{\text {box }}(\mathbf{f F})$ & $\mathbf{C}_{\text {air }}(\mathbf{f F})$ & $\mathbf{f}_{\mathbf{3 d B}}(\mathbf{G H z})$ \\
\hline $\mathbf{0 V}$ & 241 & 81.8 & 1140 & 114 & 10.2 & 6.1 \\
\hline $\mathbf{- 1 V}$ & 269 & 78 & 1360 & 90 & 11.2 & 5.8 \\
\hline $\mathbf{2 V}$ & 246 & 194 & 1100 & 19 & 9.6 & 2.7 \\
\hline
\end{tabular}

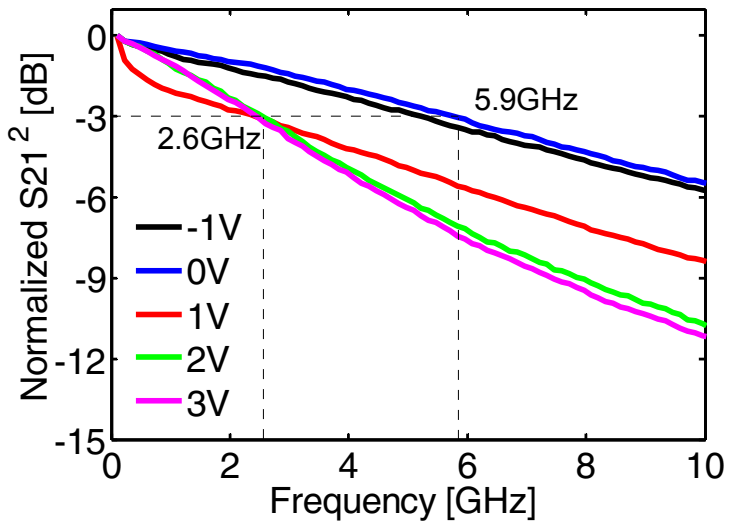

Fig. 9. Measured and normalized electro-optical S21 responses of the $50 \mu \mathrm{m}$ graphene EAM at different bias voltages showing electro-optical bandwidth of $5.9 \mathrm{GHz}$ in reverse bias and $2.6 \mathrm{GHz}$ in forward bias.

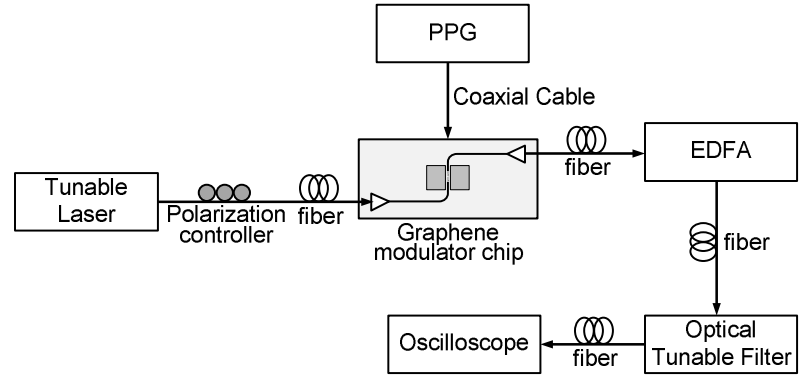

Fig. 10. Experimental setup used for high speed eye diagram measurements. $(\mathrm{PPG}=$ pseudo-random bit pattern generator, $\mathrm{EDFA}=$ erbium-doped fiber amplifier) 


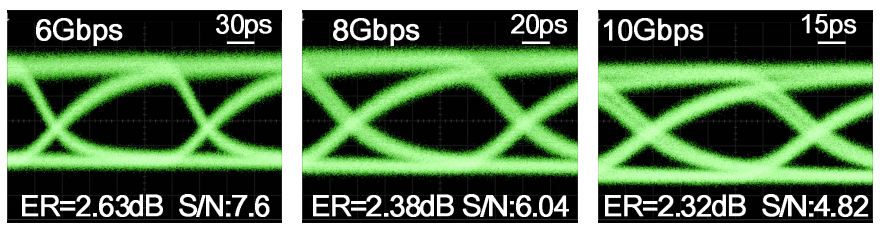

Fig. 11. Optical eye diagrams measured at $1560 \mathrm{~nm}$ for the $50 \mu \mathrm{m}$ graphene EAM device at $6 \mathrm{~Gb} / \mathrm{s}, 8 \mathrm{~Gb} / \mathrm{s}$ and $10 \mathrm{~Gb} / \mathrm{s}$ modulation speed, using a drive voltage of $2.5 \mathrm{~V}_{\mathrm{pp}}$ swing and $1.75 \mathrm{~V}$ forward bias delivered with a 50 terminated probe. Part of the noise originates from the EDFA used in the measurement setup.

\section{Conclusion}

We have reported the first compact graphene-Si electroabsorption modulator operating at $10 \mathrm{~Gb} / \mathrm{s}$ over a wide wavelength and temperature range, challenging best-in-class $\mathrm{Si}(\mathrm{Ge})$ modulators for future chip-level optical interconnects.

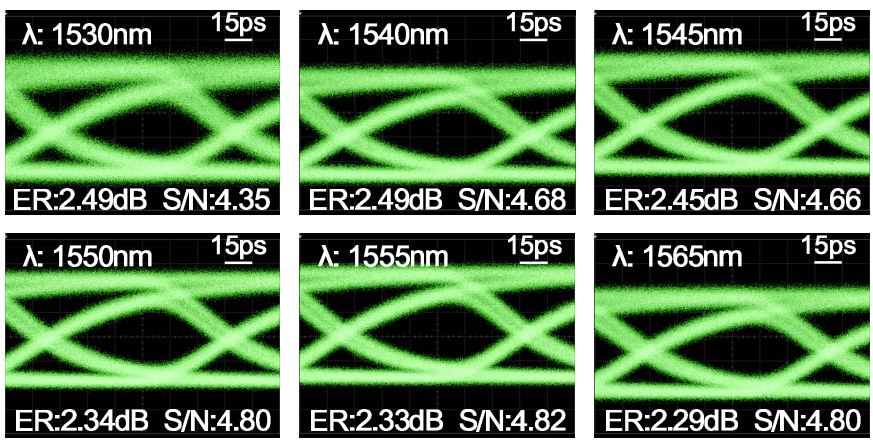

Fig. 12. Optical eye diagrams measured at $10 \mathrm{~Gb} / \mathrm{s}$ modulation speed for the $50 \mu \mathrm{m}$ graphene EAM device for wavelengths in the range $1530-1565 \mathrm{~nm}$, using a drive voltage of $2.5 \mathrm{~V}_{\mathrm{pp}}$ swing and $1.75 \mathrm{~V}$ forward bias delivered with a 50 terminated probe. A dynamic extinction ratio of $2.3 \mathrm{~dB}$ or better is obtained for all wavelengths.

Table 2. Benchmarking table comparing the performance of the presented graphene-Si EAM versus different types of state-of-the-art Si and SiGe optical modulators. The graphene EAM combines compact footprint with large optical bandwidth over a wide temperature range, with relatively low power consumption. Our work has substantially advanced the modulation speed of the graphene EAM over prior reports. Significant potential still exists to further improve the modulation speed of the graphene EAM closer to speeds obtained in highly optimized $\mathrm{Si}(\mathrm{Ge})$ modulators.

\begin{tabular}{|c|c|c|c|c|c|c|c|c|c|c|c|c|}
\hline \multirow{3}{*}{ Modulator type } & \multirow{3}{*}{ Ref. } & \multirow{2}{*}{ Footprint } & \multirow{2}{*}{ Wavelength } & \multirow{2}{*}{$\begin{array}{c}\text { Drive } \\
\text { voltage }\end{array}$} & \multirow{2}{*}{\begin{tabular}{|c|} 
Optical \\
bandwidth
\end{tabular}} & \multirow{2}{*}{\begin{tabular}{|c|}
$\begin{array}{c}\text { Temperature } \\
\text { range }\end{array}$ \\
\end{tabular}} & \multirow{2}{*}{ ER } & \multirow{2}{*}{\begin{tabular}{|c|}
$\begin{array}{c}\text { Insertion } \\
\text { Loss }\end{array}$ \\
\end{tabular}} & \multicolumn{2}{|c|}{ Power } & \multirow{2}{*}{$\begin{array}{c}3 \mathrm{~dB} \\
\text { frequency }\end{array}$} & \multirow{2}{*}{$\begin{array}{c}\text { Maximum } \\
\text { Bit Rate }\end{array}$} \\
\hline & & & & & & & & & Static & Dynamic & & \\
\hline & & {$\left[\mu \mathrm{m}^{2}\right]$} & {$[\mathrm{nm}]$} & {$[\mathrm{V}]$} & {$[\mathrm{nm}]$} & \begin{tabular}{|c|}
{$\left[{ }^{\circ} \mathrm{C}\right]$} \\
\end{tabular} & [dB] & {$[\mathrm{dB}]$} & {$[\mathrm{mW}]$} & {$[\mathrm{fJ} / \mathrm{bit}]$} & {$[\mathrm{GHz}]$} & {$[\mathrm{Gb} / \mathrm{s}]$} \\
\hline $\begin{array}{l}\text { Si Mach-Zehnder } \\
\text { Modulator }\end{array}$ & [3] & $\sim 3000 \times 500$ & 1300 & 1.5 & $>80^{*}$ & $>80^{*}$ & 3.4 & 7.1 & $\sim 20^{\#}$ & 450 & 30 & 50 \\
\hline Si Ring Modulator & [4] & $\sim 10 \times 10$ & 1550 & 0.5 & $<0.1$ & $<1$ & 6.4 & 1.2 & $<0.01$ & $\sim 1$ & 21 & 44 \\
\hline SiGe EAM & [5] & $\sim 55 \times 10$ & 1550 & 2.8 & $\begin{array}{l}35 \\
5^{\S}\end{array}$ & $\begin{array}{l}<1 \\
<40^{\S}\end{array}$ & $\sim 5$ & $\sim 4$ & $>2.2$ & 60 & $>30$ & 28 \\
\hline Graphene-Si EAM & [6] & $\sim 40 \times 10$ & 1500 & 3 & $>180$ & $n / a$ & 2.4 & n/a & $\mathrm{n} / \mathrm{a}$ & $n / a$ & 1.2 & $n / a$ \\
\hline Graphene-Si EAM & \begin{tabular}{|l|} 
This \\
work
\end{tabular} & $\sim 50 \times 10$ & 1550 & 2.5 & $>80$ & $>29$ & 2.5 & $<4$ & $<1 \times 10^{-4}$ & 350 & $2.6-5.9$ & 10 \\
\hline
\end{tabular}

"Assuming balanced Mach-Zehnder modulator with broadband 3dB splitters, "Mach-Zehnder bias control, ${ }^{8}$ Bandwidth can be traded for temperature tolerance.

\section{Acknowledgements}

This work was supported by imec's Core Partner Program. Prof B.J. Cho (KAIST, Korea) is greatly acknowledged for the generous supply of high-quality graphene.

\section{References}

[1]. G. T. Reed, G. Mashanovich, F. Y. Gardes and D. J. Thomson, "Silicon optical modulators," Nat. Photon. 4, 518-526 (2010).

[2]. I. A. Young, et al. Optical I/O Technology for Tera-Scale Computing. IEEE J. Solid-State Circuits 45.1, 235-248 (2010).

[3]. M. Streshinsky, et al, "Low power $50 \mathrm{~Gb} / \mathrm{s}$ silicon traveling wave Mach-Zehnder modulator near $1300 \mathrm{~nm}$," Opt. Express 21, 3035030357 (2013).
[4]. E. Timurdogan, C. M. Sorace-Agaskar, J. Sun, E. S. Hosseini, A Biberman and M. R. Watts, "An ultralow power athermal silicon modulator," Nat. Commun. 5, (2014).

[5]. D. Feng, et al., "High speed GeSi electro-absorption modulator at 1550 nm wavelength on SOI waveguide," Optics Express, Vol. 20, Issue 20, pp. 22224-22232 (2012).

[6]. M. Liu, et al., "A graphene-based broadband optical modulator," Nature 474, 64-67 (2011).

[7]. M. Liu, X. Yin and X. Zhang et al., "Double-layer graphene optical modulator," Nano Lett. 12(3) (2012).

[8]. J. Song, et al., "A general method for transferring graphene onto soft surfaces," Nature Nanotechnology 8, 356-362 (2013).

[9]. F. Van Laere, et al., "Compact Focusing Grating Couplers for Siliconon-Insulator Integrated Circuits," IEEE Photon. Tech. Lett., 19(23), p.1919-1921 (2007). 